\title{
Rapid Turnover of Stereocilia Membrane Proteins: Evidence from the Trafficking and Mobility of Plasma Membrane
} $\mathrm{Ca}^{2+}$-ATPase 2

\author{
M'hamed Grati, ${ }^{1}$ Mark E. Schneider, ${ }^{2}$ Karen Lipkow, ${ }^{3}$ Emanuel E. Strehler, ${ }^{4}$ Robert J. Wenthold, ${ }^{1}$ and Bechara Kachar ${ }^{2}$ \\ Laboratories of ${ }^{1}$ Neurochemistry and ${ }^{2}$ Cellular Biology, National Institute on Deafness and Other Communication Disorders, National Institutes of Health, \\ Bethesda, Maryland, 20892, ${ }^{3}$ Department of Physiology, Development, and Neuroscience, University of Cambridge, Cambridge CB2 3DY, United Kingdom, \\ and ${ }^{4}$ Biochemistry and Molecular Biology, Mayo Clinic College of Medicine, Rochester, Minnesota 55905
}

We studied the spatial distribution, mobility, and trafficking of plasma membrane $\mathrm{Ca}^{2+}$ ATPase-2 (PMCA2), a protein enriched in the hair cell apical membrane and essential for hair cell function. Using immunofluorescence, we determined that PMCA2 is enriched in the stereocilia and present at a relatively low concentration in the kinocilium and in the remaining apical membrane. Using an antibody to the extracellular domain of PMCA2 as a probe, we observed that PMCA2 diffuses laterally from the stereocilia membrane and is internalized at the apical cell border maintaining an estimated half-life of residency in the stereocilia of $\sim 5-7 \mathrm{~h}$. A computer simulation of our data indicates that PMCA2 has an estimated global diffusion coefficient of $0.01-0.005 \mathrm{\mu m}^{2} / \mathrm{s}$. Using a green fluorescent protein tag, we observed that PMCA2 is rapidly delivered to the apical cell border from where it diffuses to the entire stereocilia surface. Fluorescence recovery after photobleaching experiments show that $\sim 60 \%$ of PMCA2 in the stereocilia exhibit high mobility with a diffusion coefficient of $0.1-0.2 \mu \mathrm{m}^{2} / \mathrm{s}$, whereas the remaining pool represents a relatively immobile fraction. These results suggest that PMCA2 molecules maintain transient interactions with other components of the stereocilia, and the mobile pool of PMCA2 mediates the exchange between the stereocilia and the removal and delivery sites at the periphery of the apical cell surface. This rapid turnover of a major stereocilia membrane protein matches the previously described rapid turnover of proteins of the stereocilia actin core, further demonstrating that these organelles undergo rapid continuous renewal.

Key words: PMCA2; hair cells; endocytosis; hearing; membrane protein mobility; stereocilia

\section{Introduction}

Stereocilia are specialized membrane protrusions with actin cores on the apical surface of the sensory cells of the inner ear. Stereocilia play a key role in the process of mechanical to electrical transduction, and damage to stereocilia from trauma, such as loud noise, can result in temporary or permanent hearing loss (Gao et al., 1992). The recovery of stereocilia from moderate noise damage suggests that they have a mechanism to turnover and replace their components. We have shown previously that the stereocilia actin core undergoes continuous turnover and length regulation (Schneider et al., 2002; Rzadzinska et al., 2004; Lin et al., 2005). The dynamic length regulation of the actin core must be accompanied by a dynamic regulation of the surrounding stereocilia membrane, because these two structures maintain

\section{Received Jan. 30, 2006; revised April 21, 2006; accepted April 23, 2006.}

This work was supported by the National Institute on Deafness and Other Communication Disorders (NIDCD) Intramural Research Program. E.E.S. was supported by National Institutes of Health (NIH) Grant GM28835. K.L. was supported by NIH-National Institute of General Medical Sciences Grant GM64713. We thank Gavin P. Riordan and Saeeda Latham (NIH-NIDCD) for help with experiments and Drs. Kuni Iwasa and Ronald Petralia (NIH-NIDCD) for comments on this manuscript.

Correspondence should be addressed to Dr. Bechara Kachar, Laboratory of Cellular Biology, National Institute on Deafness and Other Communication Disorders, National Institutes of Health, 50 South Drive, Room 4249, Bethesda, MD 20892-8027.E-mail: kacharb@nided.nih.gov.

DOI:10.1523/JNEUROSCI.1215-06.2006

Copyright $\odot 2006$ Society for Neuroscience $\quad$ 0270-6474/06/266386-10\$15.00/0 a very close structural relationship (Hackney and Furness, 1995; Kachar et al., 2000). Little is known about how membrane proteins, necessary for mechanotransduction, overall shape, and viability of stereocilia, are added to and removed from the stereocilia membrane. Some proteins are preferentially enriched in stereocilia, such as the plasma membrane $\mathrm{Ca}^{2+}$-ATPase isoform 2 (PMCA2) (Dumont et al., 2001). Others, like the mechanoelectrical transduction (MET) channel and the lateral links, are maintained at specific places along stereocilia and are presumed to interact directly with the actin core (Adato et al., 2005; LeMasurier and Gillespie, 2005).

The trafficking of membrane proteins to and from stereocilia membranes requires a specialized mechanism to maintain the dynamic structure and unique organization of stereocilia. The dense actin network that makes up the stereocilia core and the cuticular plate, in which stereocilia are inserted (DeRosier and Tilney, 1989; Jaeger et al., 1994), prevents vesicles of the membrane trafficking machinery from reaching the overlaying plasma membrane (Kachar et al., 1997). The limitations to local vesicular traffic imposed by the underlying dense actin matrices and the unique distribution of membrane proteins necessary for stereocilia function raise important questions about lateral mobility and trafficking of the membrane proteins.

To characterize the specialized mechanism of membrane pro- 
tein turnover in stereocilia, we focused on PMCA2, likely the most abundant stereocilia membrane protein (Yamoah et al., 1998). PMCA2 is involved in rapid clearance of $\mathrm{Ca}^{2+}$ that enters stereocilia through the mechanoelectrical transduction channel (Lumpkin and Hudspeth, 1998) and is essential for signal adaptation (Wu et al., 1999), endolymph $\mathrm{Ca}^{2+}$ concentration (Wood et al., 2004), and stereocilia viability (Street et al., 1998; Kozel et al., 2002). In the present study, we evaluated the spatial distribution, mobility, and trafficking of PMCA2 in the apical plasma membrane of rat inner ear hair cells using a combination of immunocytochemistry and green fluorescent protein (GFP)-tagged protein expression. Our results show that PMCA2 molecules maintain an average half-life of residency time in stereocilia of $\sim 5-7 \mathrm{~h}$ and are present in two pools with distinct mobilities. A highly mobile fraction can diffuse rapidly within the membrane and plays a role in delivery and removal of PMCA2, whereas a more stable fraction may be responsible for maintaining a high concentration of PMCA2 in stereocilia.

\section{Materials and Methods}

Antibodies. PB590 and PB405 antibodies were generated by Princeton Biomolecules (Langhorne, PA) against peptides corresponding to amino acids 5-22 (TNSDFYSKNQRNESSHGG) and 135-148 (ATAQGGAEDEGEAE) of rat PMCA2 (GenBank accession number P11506), respectively. PMCA2 amino acids 135-148 are located in the first extracellular loop of the protein. The affinity purification was performed using their respective peptides immobilized on SulfoLink Coupling Gel (Pierce, Woburn, MA). The other antibodies were obtained as follows: the monoclonal antibody that recognizes all PMCAs (clone 5F10) from Axxorra (San Diego, CA); the monoclonal antibody to $\alpha$-adaptin (clone AP6) from Affinity BioReagents (Golden, CO); the monoclonal anti- $\beta$ tubulin (clone D66) and the DNA stain 4' ${ }^{\prime}$-6-diamidino-2-phenylindole (DAPI) from Sigma (St. Louis, MO); the anti-hemagglutinin (HA) antibody from Santa Cruz Biotechnology (Santa Cruz, CA); and the secondary antibodies coupled to Alexa Fluor and the rhodamine-phalloidin from Invitrogen (San Diego, CA).

Plasmid DNA constructs. Full-length cDNA encoding the PMCA2wa isoform (1199 amino acids long) expressed in rat inner ear sensory epithelia was obtained from total mRNA, prepared from postnatal day 2-15 rat vestibular neuroepithelia and organ of Corti sensory patches using Trizol (Invitrogen). Gene-specific reverse transcription was performed on DNAase-treated mRNA using the primer 5'-GGGCCTCAGCTAAAGCG-3' and Moloney murine leukemia virus reverse transcriptase (Invitrogen), followed by a PCR reaction with the forward primer 5' -ATGTGAAGCTTGGGTGATATGACCAACAGCGACTTTTAC-3' and the reverse primer $5^{\prime}$-GCAAGGTACCCTAGCCCTGCCCAGCTGC-3' using High-Fidelity Taq-Polymerase (Invitrogen). The cDNA was ligated into the HindIII and KpnI sites of the pEGFP-C2 expression vector (Clontech, Mountain View, CA). The cDNA sequence was verified by sequencing. The enhanced GFP (GFP)-tagged PMCA2wb-encoding construct has been described previously (Chicka and Strehler, 2003), and HA-tagged $\mu 2$ (AP50) and GFP-tagged $\alpha$-adaptin constructs were gifts from Dr. Juan S. Bonifacino [National Institutes of Health (NIH), Bethesda, MD].

Immunofluorescence staining. Three- to 6-week-old rats were anesthetized using $\mathrm{CO}_{2}$ and decapitated according to $\mathrm{NIH}$ guidelines (ASP1139-03 and ASP1167-02). Cochleae were freshly dissected at room temperature (RT) in Leibovitz L-15 medium (Invitrogen), fixed with 4\% paraformaldehyde (PFA) in PBS, pH 7.2, injected into the membranous labyrinth through the round and oval windows, and fixed for $30 \mathrm{~min}$ at RT. Cochleae were washed in PBS, the bony capsule and supporting tissues were removed, and organ of Corti pieces were isolated from different turns of the cochlea. These pieces were permeabilized with $0.5 \%$ Triton X-100 for $15 \mathrm{~min}$, washed three times, and blocked in 10\% fetal bovine serum-PBS for between $30 \mathrm{~min}$ and overnight. Primary antibodies (at a concentration of $\sim 1 \mu \mathrm{g} / \mathrm{ml}$ ) were applied for $1-2 \mathrm{~h}$ at RT, washed three times with PBS, and stained using fluorophore-coupled secondary antibodies. F-actin was counterstained with rhodamine-coupled phalloi$\operatorname{din}(1 \mathrm{mU} / \mu \mathrm{l})$. Individual pieces were mounted on coverslips using Prolong Antifade mounting medium (Invitrogen). Fluorescence images were obtained with a LSM 510 confocal microscope (Zeiss, Oberkochen, Germany) or Nikon (Tokyo, Japan) Eclipse with an Ultraview (PerkinElmer, Boston, MA) using a $100 \times$ objective (numerical aperture 1.4). Three-dimensional composite images were built from stacks of confocal images using Volocity (Improvision, Coventry, UK) software. We used NIH Image J software for image processing and measurements.

Cell transfection and immunofluorescence. COS-7 cells were plated on coverslips in six-well plates at $\sim 2 \times 10^{5}$ cells per well $(35 \mathrm{~mm})$ and cultured using 10\% FBS, GlutaMAX in DMEM (Invitrogen). Cells were transfected with GFP-PMCA2 wa plasmids $(0.5 \mathrm{mg} / \mathrm{ml}$ DNA $)$ using Gene Juice (Novagen, San Diego, CA). After 24 h, cells were rinsed with PBS, fixed in $4 \%$ PFA in PBS at $22^{\circ} \mathrm{C}$ for 30 min, washed three times with PBS, blocked with $4 \%$ BSA for $1 \mathrm{~h}$, incubated with primary antibodies in $4 \%$ BSA for $1 \mathrm{~h}$, washed three times in PBS, and labeled with secondary antibodies as described above. Before mounting, the cells were permeabilized and stained with $10 \mu \mathrm{g} / \mathrm{ml}$ DAPI in PBS containing $0.1 \%$ Triton $\mathrm{X}-100$.

PMCA2 clearance measurements within stereocilia. Organotypic cultures of rat sensory epithelia were prepared as described previously (Rzadzinska et al., 2004). Postnatal day 0-4 rat pups were anesthetized using $\mathrm{CO}_{2}$ and decapitated according to NIH guidelines (ASP1139-03), and their temporal bones were isolated and placed into Leibovitz L-15 media. The organ of Corti, split into two to four pieces for culturing, were attached to CellTak (BD Biosciences, Franklin Lake, NJ)-coated coverslips in culture dishes (MatTek, Ashland, MA). Cultures were incubated at $37^{\circ} \mathrm{C}$ and $5 \% \mathrm{CO}_{2}$ in DMEM F- 12 media supplemented with $5 \%$ fetal bovine serum containing $10 \mu \mathrm{g} / \mathrm{ml}$ ampicillin (Invitrogen). Explants were maintained in culture for $1-7 \mathrm{~d}$. PMCA2 clearance measurements were performed in organ of Corti hair cells to benefit from the higher consistency in shape and uniformity in lengths of the stereocilia bundles. For the clearance experiments, PB405 antibody was diluted in serum-free medium to $2 \mu \mathrm{g} / \mathrm{ml}$ and applied to the organ of Corti explants for $1-2 \mathrm{~h}$ at $4^{\circ} \mathrm{C}$. The excess antibody was removed, and the explants were washed with cold serum-free medium. The medium was exchanged for standard culture medium at $37^{\circ} \mathrm{C}$ for internalization for $1-24 \mathrm{~h}$. The specimens were then fixed with $4 \%$ PFA, permeabilized with $0.5 \%$ Triton X-100, incubated with fluorophore-coupled secondary antibodies, counterstained for actin with rhodamine-coupled phalloidin and mounted on coverslips with Prolong antifade mounting medium. The intensity of fluorescent complexes was measured either within a fixed region of interest in images of isolated stereocilia and background subtracted or within a region of interest encompassing the hair bundle with background subtracted. For each time point, three to seven explants were used, and five high-resolution frames of hair bundles were measured. Subsequently, individual stereocilia were identified, a region of interest was measured, and the data were pooled to generate the clearance curve. The clearance curves were fit with a single exponential equation, and the half-life time was calculated analytically using Origin version 7.5 (Microcal, Northampton, MA).

Hair cell transfection. Organ of Corti and vestibular hair cells of cultured explants were transfected with $1 \mu \mathrm{m}$ gold particles, coated with plasmid DNA preparations encoding GFP-PMCA2wa, GFPPMCA2wb, HA-tagged $\mu 2$, or GFP-tagged $\alpha$-adaptin, using a Helios Gene Gun (Bio-Rad, Hercules, CA). Explants were washed with PBS and fixed with $4 \%$ PFA in PBS within $6-24$ h of transfection. Hair cell structure was visualized after permeabilization with $0.5 \%$ Triton X-100 in PBS and counterstaining of F-actin with rhodamine-phalloidin for $30 \mathrm{~min}$. Those samples transfected with the construct encoding HA-tagged $\mu 2$ were processed for immunocytochemistry.

Photobleaching experiments. The fluorescence recovery after photobleaching (FRAP) experiments proved to be feasible in the longer stereocilia of the vestibular hair cells. Saccular or utricular hair cells were transfected with GFP-PMCA2wa or GFP-PMCA2wb isoforms for $24 \mathrm{~h}$. The explants were carefully removed from the cover glass, rinsed in L-15 medium without phenol red, and transferred to a sealed chamber using a grade 0 cover glass at RT. PMCA2-expressing cells were examined for 
hair bundles that were fortuitously oriented in the $x$ and $y$ planes and imaged within 15 min of being removed from the $37^{\circ} \mathrm{C}$ chamber. A beam spot of $\sim 1.2 \mu \mathrm{m}$ diameter produced with a 488 $\mathrm{nm}$ laser was positioned over the hair bundle before bleaching a portion of the stereocilia in the bundle. A preexposure frame was collected, and sequential frames after the bleaching pulse were collected during the recovery. A region of interest within the stereocilia bleach area was selected, and the average fluorescence intensity was measured for each time point. Background fluorescence was measured and subtracted from these values, and they were corrected for the fluorescence fading during image acquisition using, as reference, the fluorescence intensity in a neighboring nonphotobleached region of the hair cell. The recovery after bleaching was fit using Origin: $I(t)=A\left(1-e^{\tau \times t}\right)+C$, where $I$ is the fluorescence intensity, $A$ is the end value of the recovered intensity, $\tau$ is the fitted parameter, $t$ is the time after the bleaching pulse, and $C$ is the initial fluorescence intensity.

Simulation of diffusion of PMCA2 in the stereocilia membrane. The Smoldyn program is available from http://sahara.lbl.gov/ sandrews/ software.html with a description of the algorithm (see also http://www.pdn.cam.ac.uk/ groups/comp-cell/Smoldyn.html). A detailed account of the theory and assumptions underlying Smoldyn has been given previously (Andrews and Bray, 2004). Simulations were run on an Apple Computers (Cupertino, CA) Power Mac G5 (dual, 2 GHz). Briefly, molecules were placed within the spatially defined framework of the simulation area, and their Brownian dynamic behavior was simulated. At regular intervals $(0.1 \mathrm{~ms})$, all mobile molecules undergo a diffusive step in a random direction by a distance calculated from Fick's law. The membrane of the cylindrical stereocilia was modeled as a two-dimensional surface of area and shape equivalent to an unwrapped cylinder $10 \mu \mathrm{m}$ long and $330 \mathrm{~nm}$ in diameter. Longitudinal boundaries are periodic, i.e., molecules that leave the simulated area on the left are placed back in on the right. The narrow ankle region near the base of stereocilia was modeled by using two rectangular exclusion boxes to simulate the narrow constriction (see Fig. $2 i, j$ ). We placed a sink at the base of the simulated area below the constriction to simulate the region in which the proteins escaped the stereocilia membrane and left their neighborhood. The membrane was initially seeded with $2000 / \mu \mathrm{m}^{2}$ of freely diffusing PMCA2 monomers simulated as points within the stereocilia membrane. PMCA2 molecules were initially assigned random locations and a specific diffusion coefficient (ranging from 0.001 to $1 \mu \mathrm{m}^{2} / \mathrm{s}$ ). For the computation of protein density over time, we took a time course series of snapshots of the simulation output with Grab (Apple Computers). To quantify the simulated data, we determined the density of molecules in the time course simulations by measuring the average pixel intensity in a region of interest equivalent to the one used in the actual experiments using the NIH Image J program. The clearance curves were fit with a single-exponential equation, and the half-life time was calculated analytically using Origin. A similar procedure was used to simulate the behavior of PMCA2 molecules in the FRAP experiment (see Fig. 4).

Tissue preparation for electron microscopy. Unfixed guinea pig and mouse organs of Corti were finely dissected, gently positioned onto fastfreezing specimen holders, quick frozen by contact with a liquid nitrogen-cooled sapphire block of a quick-freezing machine, and promptly transferred to liquid nitrogen. Frozen samples were freeze substituted in $1.5 \%$ uranyl acetate in absolute methanol at $-90^{\circ} \mathrm{C}$, infiltrated with Lowicryl $\mathrm{HM}-20$ resin at $-45^{\circ} \mathrm{C}$, and polymerized with UV light. Freeze-fracture and freeze-etching replicas were performed as described previously (Kachar et al., 2000). Thin sections and replicas were viewed and photographed with a transmission electron microscope equipped with an energy filter.

\section{Results}

\section{PMCA2 is enriched and uniformly distributed along the} stereocilia membrane

To characterize the steady-state distribution of PMCA2 along stereocilia and the contiguous apical membrane overlaying the cuticular plate, we measured and analyzed the pattern of distribution of fluorescence intensity in immunofluorescently labeled, freshly dissected, or cultured rat organ of Corti and vestibular hair cells. We used two affinity-purified antibodies specific for PMCA2 and a monoclonal antibody that labels all PMCA isoforms. All three antibodies produced similar results in outer hair cells (OHCs) of the organ of Corti and vestibular hair cells of the sacculae, utricle, and ampula. Figure 1 shows representative fluorescence confocal images of OHCs and vestibular hair cells labeled with the 5F10 monoclonal and PB590 polyclonal antibodies. By measuring the values of relative pixel intensity (rpi) in confocal images of the OHCs, we observed that fluorescence intensity was constant across stereocilia of the same rank in a bundle (Fig. $1 b$ ) and along the entire length of each individual stereocilium (Fig. 1c). We also observed that fluorescence intensity was constant over areas of the apical membrane overlaying the cuticular plate near the stereocilia (Fig. 1b). The average values for the rpi shown in Figure $1 b$ were $191.8 \pm 19.0(n=7)$ for the stereocilia membrane, $89.8 \pm 4.1(n=5)$ for the region of the apical membrane around the base of the stereocilia and over the 
cuticular plate, and 57.9 $\pm 2.5(n=5)$ for the nearby background. When normalized for the background, the fluorescence over stereocilia was 133.9 and over the apical membrane was 31.9. Considering that the measured fluorescence intensity over the stereocilia corresponds to the integrated fluorescence of a series of 300 -nm-diameter cylinders separated by $60 \mathrm{~nm}$ interstereociliary separations (estimated from electron microscopy images; data not shown), we estimate that the relative fluorescence intensity for the unwrapped stereocilia membrane is 51.2 . This corresponds to 1.6 times the fluorescence of the apical membrane measured over the cuticular plate region.

The hair bundle of vestibular hair cells is characteristically longer than the hair bundle of organ of Corti hair cells and also contains a single microtubule-based true cilium per bundle, called a kinocilium. Figure $1 d$ shows a vestibular hair bundle that was splayed to allow visualization of individual stereocilia. A typical intact hair bundle is shown in the inset of the same figure. The measured values of PMCA2 immunofluorescence over long and short stereocilia were relatively constant, whereas the PMCA2 immunofluorescence values along the kinocilium membrane were just slightly above the background levels. These results suggest that PMCA2 is evenly distributed along all stereocilia but expressed at very low levels in the kinocilium membrane. The relatively constant fluorescence intensity measured for stereocilia of different lengths in the bundle suggested that all stereocilia in each bundle have a constant density of PMCA2.

\section{Removal and internalization of PMCA2 from stereocilia}

We evaluated the lateral mobility of stereocilia membrane proteins by characterizing the removal of constitutively expressed PMCA2 from stereocilia using a polyclonal antibody (PB405) raised against a polypeptide with the sequence matching the first extracellular loop of PMCA2. The antibody was affinity purified and tested in an immunocytochemistry assay for its ability to label the surface of nonpermeabilized GFP-PMCA2-transfected COS-7 cells (Fig. 2a) and hair cell stereocilia (data not shown). Preincubation of the antibody with an excess of the immunizing peptide showed no labeling of stereocilia (Fig. $2 b$ ). For the removal and internalization experiments, organ of Corti explants were incubated with the PB405 antibody for $1 \mathrm{~h}$ at $4^{\circ} \mathrm{C}$, followed by washing out of unbound antibody and then internalization by raising the temperature to $37^{\circ} \mathrm{C}$. Samples were fixed at successive time intervals, and the PMCA2-antibody complex was labeled with a secondary fluorescent-labeled antibody, and the actin filaments were counterstained with rhodamine-phalloidin. The PB405-PMCA2 complexes were maintained in stereocilia membranes when membrane trafficking was inhibited by holding the tissue at $4^{\circ} \mathrm{C}$ for up to $24 \mathrm{~h}$ (Fig. $2 c, d$ ). When the temperature was raised to $37^{\circ} \mathrm{C}$, the fluorescence intensity along the stereocilia decreased progressively (Fig. $2 d-f$ ). We measured the changes in relative fluorescence intensity as a function of time for two types of region of interest, one covering the entire stereocilia bundle and the other covering a portion of individual stereocilia as shown (Fig. 2d-f). The relative fluorescence intensity corresponding to the PMCA2-antibody complexes decayed progressively for both stereocilia and the hair bundle compartment. The data were fitted to a single-exponential equation, and the $t_{1 / 2}$ was determined (Fig. $2 h$ ). The individual stereocilia clearance was $t_{1 / 2}$ $=5.2 \mathrm{~h}$, whereas the entire bundle clearance was $t_{1 / 2}=7.3 \mathrm{~h}$. The slower rate measured for the entire bundle was likely attributable to the formation of some complexes at the region of the interstereociliary links and was visualized as bright fluorescence puncta (Fig. $2 e, f$, arrows). These puncta persisted beyond $24 \mathrm{~h}$ when the fluorescence along the rest of the stereocilia was virtually cleared. Concurrently with the progressive reduction of stereocilia antibody-PMCA2 fluorescence, a pattern of vesicles and fluorescence puncta emerged in the apical cytoplasm around the cuticular plate. This internalized fluorescence could be detected within $1 \mathrm{~h}$ of incubation at $37^{\circ} \mathrm{C}$ (Fig. $2 g$ ) and increased in intensity over time. The fluorescence in the cytoplasm was consistently limited to the pericuticular and subcuticular regions of the cytoplasm and never reached the basal or synaptic region of the cell (data not shown).

To compare our experimental data with a diffusion-based process, we used a recently developed program called Smoldyn (Andrews and Bray, 2004) to model the diffusion of PMCA2 while taking into account the $2000 / \mu \mathrm{m}^{2}$ density of the protein and the geometry of the stereocilia membrane. The narrow cylindrical shape and tapered base of each stereocilium and its structured membrane impose peculiar constraints to diffusive behavior of the membrane proteins and complicate interpretation of our data. The Smoldyn program uses an approach known as Brownian dynamics to accurately capture stochastic behavior and simulate diffusive gradients in compartments of modeled configuration (Lipkow et al., 2005). We modeled the tubular stereocilia membrane as an unfolded rectangular surface, with length and width corresponding to the length and the circumference, and an additional steric constraint to represent the tapered base of the stereocilia, as illustrated in the output from a typical Smoldyn simulation (Fig. 2i,j). To evaluate the global diffusion rate associated with clearance of PMCA2, we compared our experimental data shown in Figure $2 h$ with data obtained when we simulate the clearance process using diffusion rates from 0.0001 to $0.1 \mu \mathrm{m}^{2} / \mathrm{s}$. We observed that our experimental clearance data fit between simulated diffusion rates of $0.01-0.005 \mu \mathrm{m}^{2} / \mathrm{s}$ (Fig. $2 k$ ). For our estimates of diffusion coefficients, we must take into account the approximations made and especially the potential perturbations and drag effects associated with possible dimerization of PMCA2 by our antibody labeling in the clearance experiments. In an unobstructed system, the Saffman-Delbrück model predicts little difference in the diffusion coefficient for large and small proteins or dimerization of a diffusant (Saxton and Jacobson, 1997). However, in a complex environment, larger aggregates and interactions with immobile species can follow an initial dimerization process (Saxton and Jacobson, 1997). It is possible that the fluorescent puncta that we observed accumulating near the base of the stereocilia in our clearance experiments (Fig. 2e,f) correspond to larger complexes of antibody-PMCA2 molecules with immobile structures near the base of the stereocilia. However, the puncta represent a fraction of the total fluorescence measured and had a limited effect on the estimated $t_{1 / 2}$ (Fig. $2 h$ ). We believe that the potential effects of antibody cross-linking are minimized by the fact that the antibody used is directed to a small peptide, which would limit access to a single antibody molecule and restrict cross-linking of the PMCA2 molecules.

\section{Newly synthesized PMCA2 is rapidly incorporated into the stereocilia membrane}

We further evaluated the trafficking and dynamics of PMCA2 by characterizing the reciprocal process of PMCA2 incorporation into the stereocilia membranes using GFP-tagged PMCA2. We used cDNA constructs encoding N-terminally GFP-tagged PMCA2, which have been shown previously to express protein products that correctly fold and are targeted to the plasma membrane (Chicka and Strehler, 2003). Alternative splicing can generate several isoforms of variants of PMCA2. PMCA2wa and 

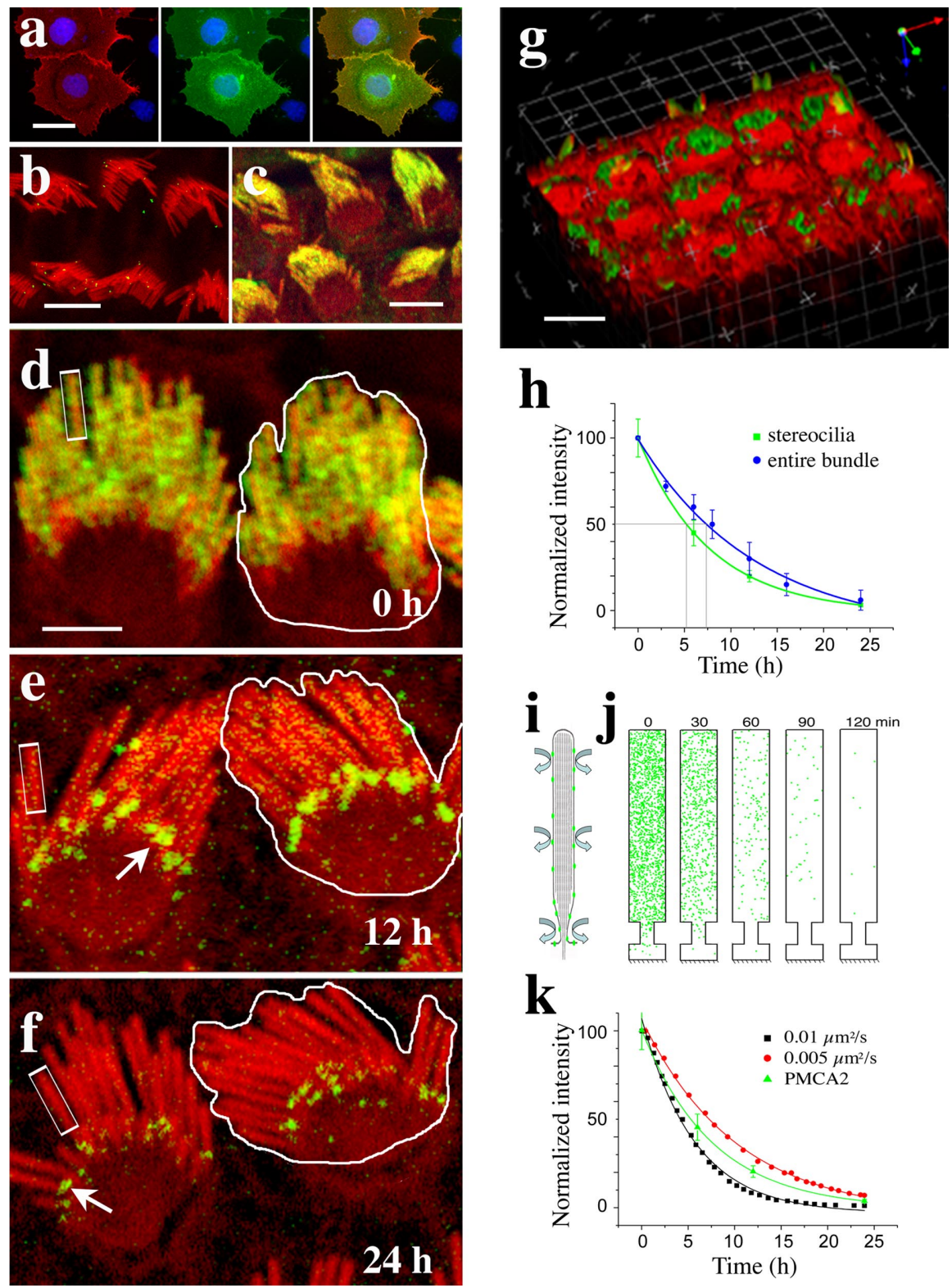

Figure 2. Removal and internalization of PMCA2 traced by antibody labeling. $\boldsymbol{a}$, Characterization of the specificity of PB405 antibody by immunofluorescence. Labeling of nonpermeabilized COS-7 cells with the PB405 antibody (red in left panel) shows colocalization with the GFP-PMCA2wa on the surface of transfected cells (green in the middle panel). The nuclei of transfected and nontransfected cells were counterstained with DAPI (blue). Panel on the right shows the overlap of the red and green fluorescence (yellow) on the surface of the transfected cells. Nontransfected cells visualized with the DAPI staining show no surface labeling. $\boldsymbol{b}$, Characterization of the specificity of the antibody in hair cells. PB405 antibody labeling (green) shows no immunoreactivity on the surface of hair cells when used in the presence of $2 \mu \mathrm{g} / \mathrm{ml}$ of the immunizing peptide. The organ of Corti explants were counterstained for actin with rhodamine-phalloidin (red). c, Demonstration of the stability of the antibodyPMCA2 binding. Control immunofluorescence experiments performed on tissues, maintained at $4^{\circ} \mathrm{C}$ for $24 \mathrm{~h}$ after the $1 \mathrm{~h}$ incubation of the tissue with PB405 antibody. The persistence of PMCA2 immunoreactivity indicates that the antibody remains bound to the PMCA2 for the duration of our clearance experiments. The organ of Corti explants were counterstained for actin with rhodamine-phalloidin (red). $\boldsymbol{d}-\boldsymbol{f}$, Time course of PMCA2-antibody complex (green) clearance from the stereocilia membrane in cultured outer hair cells after $1 \mathrm{~h}$ with the antibody at $4^{\circ} \mathrm{C}$. Actin is counterstained with rhodamine-phalloidin (red). PMCA2-antibody complexes are randomly distributed within the entire hair bundle at time $0 \mathrm{~h}$. The intensity of fluorescence along individual stereocilia and along the entire bundle decreases progressively over time. White arrows point to fluorescence puncta near the base of the stereocilia. $\boldsymbol{g}$, Volume view of a stack of confocal images of the apical region of three rows of hair cells showing the internalized antibody-PMCA complexes detected as vesicles and puncta within $1 \mathrm{~h}$ in the region below the apical membrane around the cuticular plate. $\boldsymbol{h}$, Time course plots of the normalized and averaged (rpi $\pm S D$ ) relative fluorescence intensity measured over randomly selected regions of interest (rectangles in $\boldsymbol{d}-\boldsymbol{f}$ ) along individual stereocilia (excluding the fluorescence puncta, arrows in $\boldsymbol{d}$ and $\boldsymbol{e}$ ) and over the entire bundle as outlined in $\boldsymbol{d}-\boldsymbol{f}$ (including the fluorescence puncta, arrows in $\boldsymbol{d}$ and $\boldsymbol{e}$ ). The data were fitted to a single-exponential equation, and the $t_{1 / 2}$ was determined. The individual stereocilia clearance was $t_{1 / 2}=$ $5.2 \mathrm{~h}$, whereas the entire bundle clearance demonstrated a $t_{1 / 2}=7.3 \mathrm{~h} . \boldsymbol{i}, \boldsymbol{j}$, Simulation of the distribution and clearance of PMCA2 using Smoldyn. The membrane of the cylindrical stereocilia with a tapered base is unwrapped and modeled as a two-dimensional
PMCA2wb, which are two described alternative splice variants that differ within the C-terminal domain (Strehler and Zacharias, 2001), may differentially associate with scaffolding proteins (DeMarco and Strehler, 2001; DeMarco et al., 2002). In our experiments, hair cells from rat inner ear sensory tissues were transfected with the GFP-tagged constructs for both PMCA2 splice variants. Cultured organ of Corti and vestibular sensory tissues were transfected using a gene gun. Both PMCA2wa and PMCAwb produced a similar pattern and time course of fluorescence intensity distribution. The GFP-PMCA2 fluorescence rapidly distributed along the stereocilia membrane of the various hair cells, including up to $\sim 100-\mu \mathrm{m}$ long stereocilia of the ampullary hair cells. After $24-48$ h of transfection, all transfected hair cells showed GFP fluorescence along their stereocilia and over their apical membrane (Fig. $3 a-c$ ). Analyses of the incorporation into vestibular hair cells showed that both GFP-PMCA2wa and GFP-PMCA2wb are expressed at very low levels in the kinocilium compared with the stereocilia (Fig. $3 b$ ). The total fluorescence along the stereocilia consistently increased with time, reaching maximum levels at $\sim 36-48 \mathrm{~h}$ after transfection (Fig. 3c). We analyzed cultures up to $72 \mathrm{~h}$ after transfection (data not shown), and we did not detect any difference in overall morphology or shape of stereocilia between transfected and nontransfected cells. Thus, there is no evidence for an increase in surface area of the apical or stereocilia plasma membrane attributable to overexpression of PMCA2.

Early transfection times showed a consistent pattern of incorporation but a broad variation in the degree of fluorescence. GFP fluorescence was detected as early as $5-8 \mathrm{~h}$ after transfection, initially appearing at the apical border of hair cells around the cuticular plate region and as a fine speckled pattern of fluorescence in the apical membrane and along the entire stereocilia (Fig. 3d-f). The fine speckled pattern of fluorescence was replaced progressively by a homogeneous and brighter

\section{$\leftarrow$}

surface (i). An example (using arbitrary, scaled-down parameters for better visualization) of a simulation output (j) for a density of PMCA2 molecules (green dots) of $400 / \mu \mathrm{m}^{2}$, a diffusion rate of $0.1 \mu \mathrm{m}^{2} / \mathrm{s}$, and a stereocilium $5 \mu \mathrm{m}$ long shows a virtual clearance of all PMCA2 molecules from the stereocilia membrane within $2 \mathrm{~h}$. $\boldsymbol{k}$, Comparison of the experimental data shown in $\boldsymbol{h}$ to the quantification of the data obtained from the output of the simulation using diffusion rates of $0.01 \mu \mathrm{m}^{2} / \mathrm{s}$ and $0.005 \mu \mathrm{m}^{2} / \mathrm{s}$ and stereocilia length of $10 \mu \mathrm{m}$. Scale bars: $\boldsymbol{a}-\boldsymbol{c}, 10 \mu \mathrm{m} ; \boldsymbol{d}-\boldsymbol{f}, 5 \mu \mathrm{m} ; \boldsymbol{g}, 10 \mu \mathrm{m}$. 

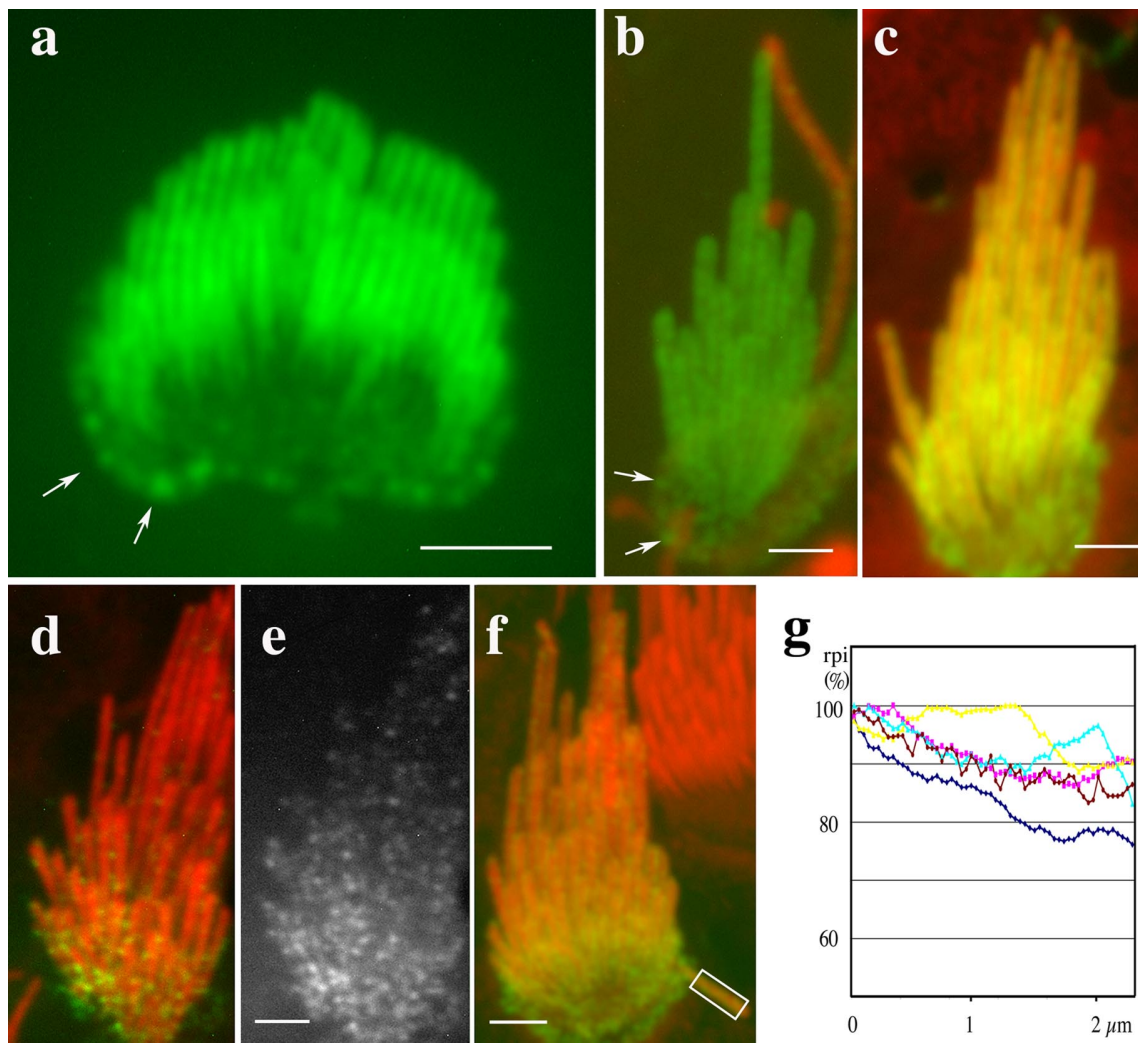

Figure 3. Expression of GFP_PMCA2wa and GFP_PMCA2wb isoforms in vestibular and organ of Corti hair cells. $\boldsymbol{a}, \boldsymbol{b}$, Confocal images of organ of Corti $(\boldsymbol{a})$ and vestibular $(\boldsymbol{b})$ hair cells expressing GFP-PMCA2wa (green) isoform after 24 h of expression showing the rapid incorporation and the uniform distribution of PMCA2 into the stereocilia. The levels of GFP-PMCA2wa in the kinocilium were much lower than in the stereocilia, as seen in $\boldsymbol{b}$, in which the hair cells were counterstained for tubulin (red). Arrows point to green fluorescent puncta around the cell border. $c$, Confocal image of a vestibular hair cell transfected for $48 \mathrm{~h}$ with GFP_PMCA2wb isoform (green) and counterstained for actin (red). $\boldsymbol{d}, \boldsymbol{e}$, Vestibular hair cell transfected with GFP_PMCA2wa (green) for $6 \mathrm{~h}$ and counterstained for actin (red). The green channel is shown separately in $\boldsymbol{e}$ to better visualize the fine speckled pattern of fluorescence that forms a gradient from the base to the tip of the stereocilia bundle. $f$, Vestibular hair cell transfected with GFP-PMCA2wa (green) for $18 \mathrm{~h}$ and counterstained for actin (red). $\boldsymbol{g}$, Plot of normalized average rpi along the stereocilia measured as indicated in the rectangular region of interest shown in $\boldsymbol{f}$ in five cells expressing either GFP_PMCA2wa or GFPPMCA2wb for $18 \mathrm{~h}$ or less shows a small but consistent fluorescence gradient from base to tip. Scale bars, $2.5 \mu \mathrm{m}$.

fluorescence over time. To characterize the distribution of GFPPMCA2 along stereocilia, we measured and plotted fluorescence intensity along stereocilia in cells transfected for $18 \mathrm{~h}$ (Fig. $3 g$ ). We observed a small but consistent fluorescence intensity gradient that decreased by $\sim 5-10 \%$ from the stereocilia base to the tip (Fig. $3 g$ ), suggesting the presence of a diffusive gradient.

\section{Characterization of lateral mobility of PMCA2 in stereocilia}

The kinetics of clearance of PMCA2 from stereocilia and delivery of GFP-PMCA2 to stereocilia suggest that a diffusion-based mechanism mediates these processes. For the evaluation of the PMCA2 lateral mobility in the stereocilia membrane, we used the FRAP technique on GFP-PMCA2-transfected cells. Twenty-four hours after transfection with either GFP-PMCA2wa or GFPPMC2wb, vestibular sensory epithelium explants were transferred to L-15 medium to maintain constant $\mathrm{pH}$ and to wash out the phenol red before placing the explants in a sealed optical chamber for confocal imaging. Experiments were performed within $15 \mathrm{~min}$ of removal from the $37^{\circ} \mathrm{C}$ incubator. For the photobleaching, we used a Gaussian-shaped laser beam focused to produce a bleaching radiation over a radius of $\sim 1.2 \mu \mathrm{m}$. We calibrated duration and intensity of the photobleaching pulse

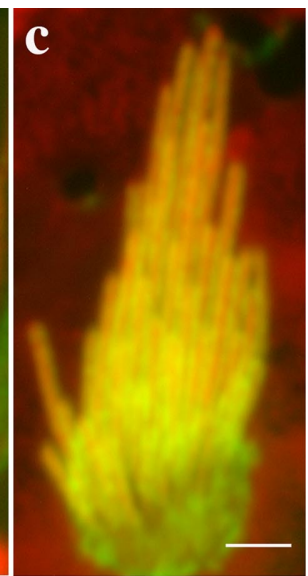

using transfected supporting cells. For each of the constructs, we were able to analyze at least three hair cells whose stereocilia were optimally oriented for imaging individual stereocilia. The initial fluorescence and subsequent recovery after the 1-2 s exposure to the photobleaching beam were recorded by acquiring a stream of images at regular 10-15 s intervals obtained with a spinning-wheel confocal microscope equipped with a highperformance camera. The average intensity of fluorescence from a $1-\mu \mathrm{m}$ long region over the bleached area of an individual stereocilium (Fig. 4a) was measured for each image, background subtracted, normalized for global loss of fluorescence during sampling, and fitted with an exponential model. For both the PMCA2 wa and PMCAwb splice forms, the fluorescence recovery reached an asymptote within 80-100 s (Fig. 4b,c). The fraction that was able to diffuse, termed the mobile fraction, corresponded to $\sim 60 \%$ of the total PMCA2 and showed a recovery profile consistent with a simple diffusionbased process. The difference between the final, asymptotic level of fluorescence and the initial, baseline level of fluorescence ( $\sim 40 \%)$ corresponds to the fraction of the PMCA2 that is unable to diffuse freely on the cell surface in the timescale of the experiment. We are limited in the characterization of fluorescence recovery because the diameter of individual vestibular hair cell stereocilia is between 200 and $400 \mathrm{~nm}$ and within the diffraction limited resolution of the fluorescence imaging. Thus, the requirements for operation at low fluorescence levels and the need for high spatial resolution forces us to compromise the temporal resolution of the data. The shortest exposure times per image were $\sim 10 \mathrm{~s}$. Moreover, the fluorescence recovery is fast, making it difficult to collect enough data points to accurately model the initial loss of fluorescence by photobleaching and the recovery function. To estimate the diffusion rate for PMCA2 in the mobile fraction, we again used the Smoldyn program. We simulated our FRAP experiments using a PMCA2 density equivalent to the mobile fraction within the geometric constraints of the stereocilia membrane and a range of diffusion rates (Fig. 4d). We found that our experimentally determined recovery profile falls between the simulated recovery profiles for the diffusion rates of 0.1 and $0.2 \mu \mathrm{m}^{2} / \mathrm{s}$ (Fig. $4 e$ ).

\section{Spatial organization of the apical membrane trafficking machinery}

The delivery and removal of PMCA2 described above occurred from a region around the cuticular plate. A previous ultrastructural study in frog vestibular hair cells (Kachar et al., 1997) identified the structural correlates of an intense vesicular traffic compartmentalized to a narrow band of cytoplasm around the cuticular plate. Using a combination of freeze-fracture and freeze-substitution techniques, we observed in the mammalian 

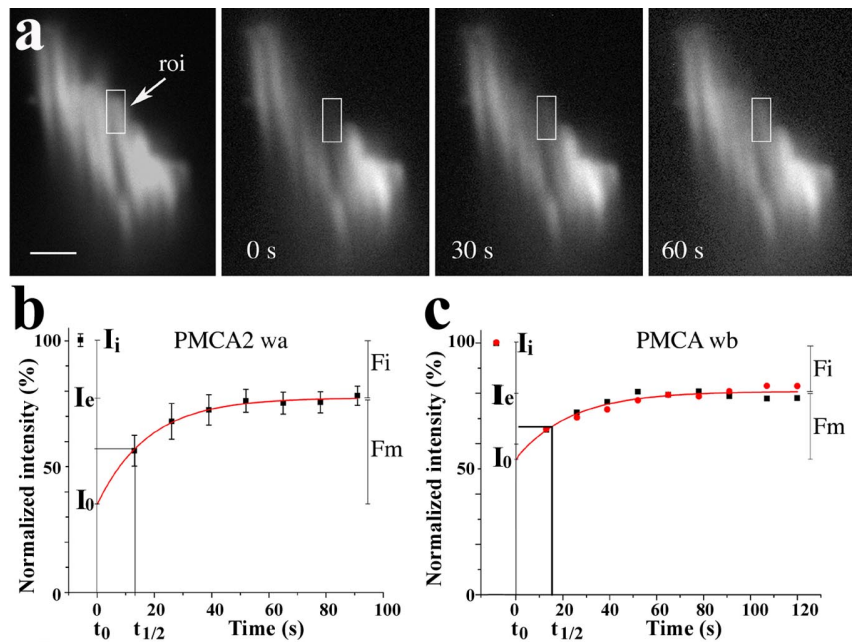

c

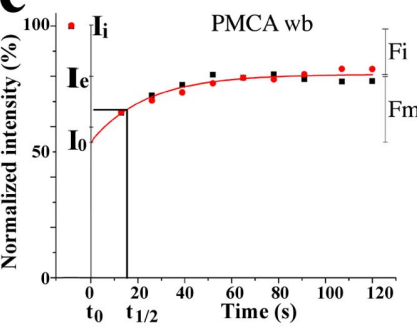

d
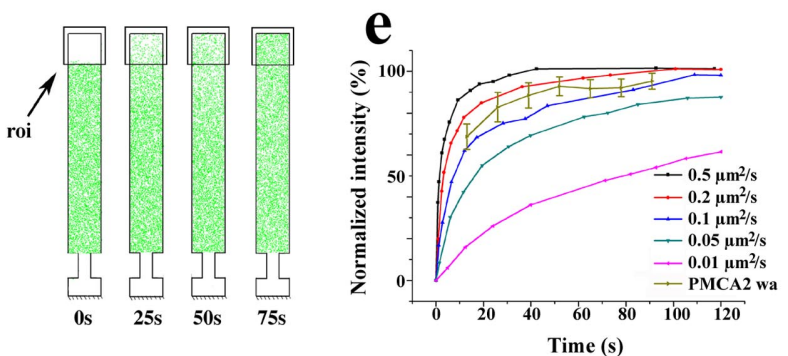

Figure 4. Mobility of GFP-PMCA2 characterized with FRAP. $\boldsymbol{a}$, Time course sequence of confocal images of the stereocilia bundle of a PMCA2wa-GFP-transfected vestibular hair cell taken before and after photobleaching a $\sim 1.2 \mu \mathrm{m}$ region at the center. $\boldsymbol{b}$, FRAP analysis of three vestibular hair cell stereocilia expressing GFP-PMCA2wa. The initial fluorescence intensity $\left(I_{i}\right)$ before photobleaching was set to $100 \%$. The average fluorescence intensity was measured over the region of interest (roi) placed over individual stereocilia within the photobleached region as shown in $\boldsymbol{a}$. The relative pixel intensity was background subtracted and normalized to the preexposure control in the region of interest. The fluorescence recovery for three stereocilia from two different hair cells was fit with a single exponential $\left(r^{2}=0.999\right)$. The mobile fraction $\left[F_{\mathrm{m}}=\left(I_{\mathrm{e}}-I_{0}\right) /\left(I_{\mathrm{i}}-I_{0}\right)\right]$ was $\sim 62 \%$ of the photobleached fraction with a half-life $\left(t_{1 / 2}\right)$ for recovery of $12.3 \mathrm{~s}$. $I_{0}$, Intensity at time point $0 ; I_{\mathrm{e}}$, end value of the recovered intensity; $F_{\mathrm{i}}$, immobile fraction $=1-F_{\mathrm{m}}$. C, FRAP analysis of two vestibular hair cell stereocilia expressing GFP-PMCA2wb. The fluorescence recovery for two hair cell stereocilia was fit with a single exponential $\left(r^{2}=0.989\right)$. The mobile fraction $\left(F_{\mathrm{m}}\right)$ comprised $\sim 58 \%$ of the bleached fraction with $t_{1 / 2}=16.4$ s for recovery. $\boldsymbol{d}$, Output of a typical simulation of the FRAP experiment using Smoldyn. We simulate our FRAP experiments using a PMCA2 density equivalent to the mobile fraction within the geometric constraints of the stereocilia membrane and a range of diffusion rates. $\boldsymbol{e}$, Plot showing the recovery profile of the simulated FRAP for recovery rates between 0.01 and $0.5 \mu \mathrm{m}^{2} / \mathrm{s}$. Our experimentally determined recovery profile shown in $\boldsymbol{b}$, when normalized to $100 \%$ to match the simulation, falls between the simulated recovery profiles for the diffusion rates of 0.1 and $0.2 \mu \mathrm{m}^{2} / \mathrm{s}$. Scale bar, $1 \mu \mathrm{m}$.

organ of Corti hair cells (Fig. 5a,b) and vestibular hair cells (data not shown) a similar compartmentalized organization of membrane organelles around the cuticular plate, indicating that this organization is a common feature for all hair cells. The freezefracture view in Figure $5 b$ shows a large area of the apical plasma membrane of an organ of Corti hair cell in which the membrane overlying the cuticular plate contains only the insertions of the stereocilia, whereas the area close to the cell border shows a population of variably sized membrane invaginations and pits. As in frog hair cells, these membrane invaginations and pits likely correspond to structural intermediates of endocytosis and exocytosis. The thin-section longitudinal view of this region (Fig. $5 a$ ) shows the cuticular plate filament network that dominates the apical region below the hair bundle and its close association with

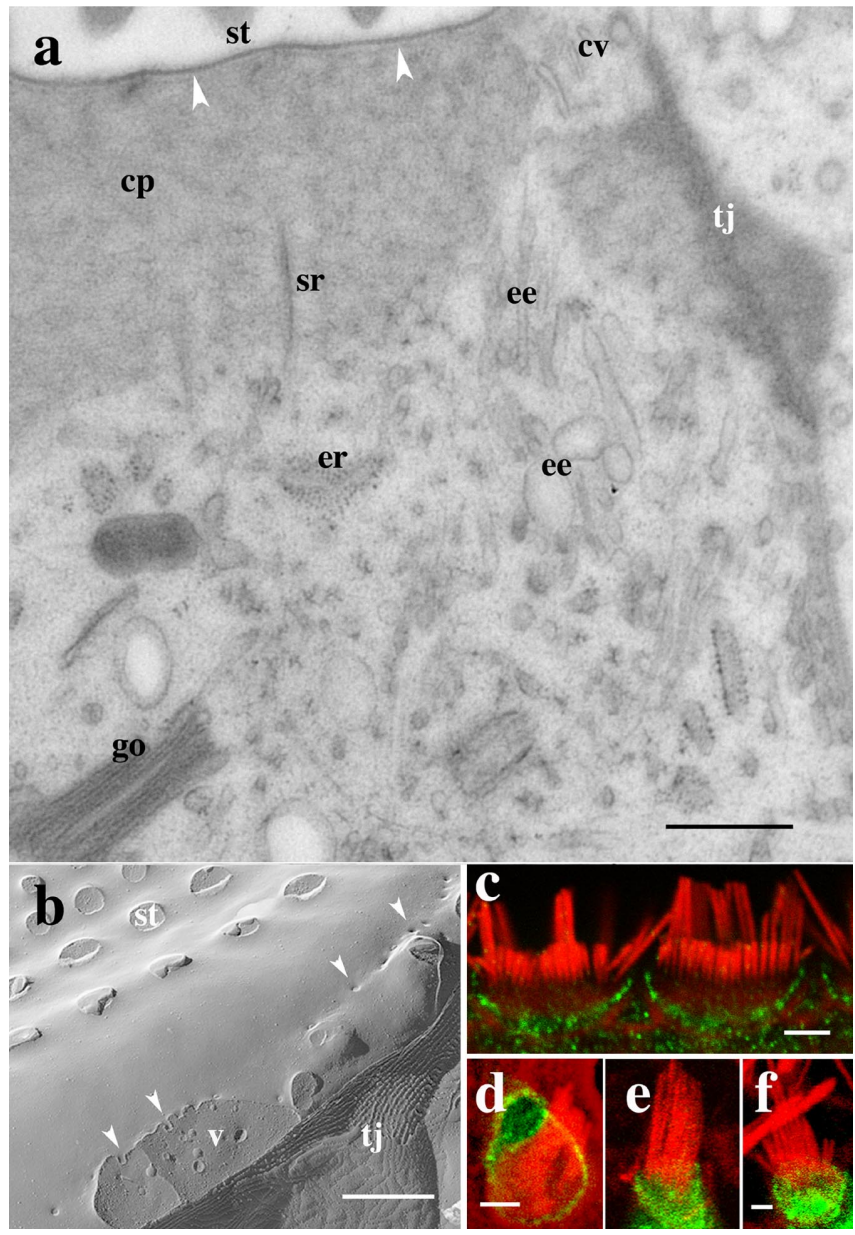

Figure 5. Spatial organization of apical membrane trafficking organelles. $\boldsymbol{a}$, Thin-section longitudinal view of the apex of an organ of Corti hair cell. The cuticular plate matrix (cp) containing the stereocilia (st) rootlets (sr), dominates the subapical membrane cytoplasm and maintains a close association with the plasma membrane (arrowheads) precluding the presence of cytoplasmic organelles. A narrow strip of cytoplasm that contains the numerous membrane-bound organelles bridges the apical membrane and the cytoplasm below the cuticular plate. The subcuticular cytoplasm contains endoplasmic reticulum (er), Golgi cysternae (go), and early endosomes (ee). Coated (cv) and uncoated small vesicles are consistently found around the cuticular plate near the tight junction (tj). $\boldsymbol{b}$, Freeze-fracture view of the apical plasma membrane of an organ of Corti hair cell in which the membrane overlying the cuticular plate shows the insertions of the stereocilia (st) and, at the cell border, near the tight junction (t), a number of variably sized membrane invaginations and pits (arrowheads). A cross-fracture of the cytoplasm below the apical membrane border shows vesicles (v). c, Confocal immunofluorescence showing that the $\alpha$-adaptin subunit of the clathrin AP2 complex (green) is immunolocalized around the apical cell border of the outer hair cells but not in the stereocilia counterstained for actin (red). $\boldsymbol{d}, \boldsymbol{e}$, Confocal images showing EGFP-tagged $\alpha$-adaptin (green) restricted to the apical cell border and excluded from the stereocilia membrane in organ of Corti (d) and vestibular (e) hair cells. $\boldsymbol{f}$, Confocal image showing immunofluorescently labeled, HAtagged $\mu 2$ subunit (green) of the AP 2 adaptor complex in a vestibular hair cell. Both $\alpha$-adaptin as well as the $\mu 2$ subunit are incorporated into the apical plasma membrane around the base of the stereocilia but are excluded from stereocilia membrane. Scale bars: $\boldsymbol{a}, \boldsymbol{b}, 0.8 \mu \mathrm{m} ; \boldsymbol{c}-\boldsymbol{f}, 1 \mu \mathrm{m}$.

the plasma membrane precluding the presence of cytoplasmic organelles. A narrow strip of cytoplasm that contains numerous membrane-bound organelles bridges the apical membrane and the cytoplasm below the cuticular plate. The subcuticular cytoplasm contains mitochondria, endoplasmic reticulum, Golgi, and endosomal vesicles. Coated and uncoated small vesicles are consistently found adjacent to tubulovesicular structures and other components of the endosomal and Golgi systems. 


\section{Exclusion of components of the clathrin complex from stereocilia}

A previous study shows that membrane pits and vesicles around the cuticular plate are clathrin coated (Kachar et al., 1997), indicating that the clathrin-based mechanism could be the principal pathway for endocytosis and membrane protein internalization in the apical membrane of hair cells. Because membrane pits and vesicular structures are excluded from stereocilia and from the cuticular plate region and are found only near the border of the apical surface, we wanted to determine whether the earlier steps of the clathrin-mediated internalization are also excluded from the stereocilia. We determined by immunofluorescence the distribution of the $\alpha$-adaptin and $\mu 2$ subunits of the AP2 adaptor complex, which is involved in binding cargo proteins in the plasma membrane for clathrin-mediated endocytosis. $\alpha$-Adaptin is an AP2 complex-specific subunit responsible for the anchoring of AP2 to the plasma membrane, and $\mu 2$ binds $\mathrm{Yxx} \varnothing$ motifs on cargo proteins (Nakatsu and Ohno, 2003). We observed immunofluorescence for $\alpha$-adaptin (Fig. $5 c$ ) and $\mu 2$ (data not shown) subunits around and below the cuticular plate but not on the stereocilia and kinocilia membranes. To obtain additional evidence for the absence of AP2 components from the plasma membrane of stereocilia, we overexpressed GFP-tagged $\alpha$-adaptin and HA-tagged $\mu 2$ in cultured hair cells. Both GFP-tagged $\alpha$-adaptin (Fig. $5 d, e$ ) and $\mu 2$ (Fig. $5 f$ ) showed a distribution similar to that obtained for their respective native proteins observed using immunofluorescence with no detectable fluorescence in stereocilia or kinocilia.

\section{Discussion}

Cellular protrusions that are not directly accessible to synthetic and trafficking machineries of the cell require specialized mechanisms for recruitment, assembly, maintenance, and turnover of their components. Stereocilia are one such example in which elaborate protein ensembles are maintained at distances that can be up to $100 \mu \mathrm{m}$ from their sites of delivery and uptake. In the present report, we characterize the distribution, lateral mobility, and turnover of PMCA2, an essential protein for stereocilia function. Our results suggest that a pool of PMCA2 with high lateral mobility mediates rapid distribution of PMCA2 to and from stereocilia via peripheral sites at the margin of the hair cell. We also propose that a pool of PMCA2 with restricted mobility is responsible for enrichment of PMCA2 in the stereocilia membrane. Furthermore, we consider how rapid lateral diffusion allows sites of membrane exocytosis and endocytosis to coexist at the margins of the hair cell and avoid futile cycling of PMCA2.

\section{PMCA2 is enriched and uniformly distributed in the stereocilia}

Our immunofluorescence measurements show that, at steady state, the distribution of PMCA2 in the outer hair cell stereocilia is 1.6-fold higher than in the membrane over the cuticular plate. Interestingly, at steady state, the distribution of PMCA2 along the entire stereocilia length is uniform and is constant among stereocilia of different heights in the same bundle for both the organ of Corti and vestibular hair cells. The main function of PMCA2 is to remove calcium entering the stereocilia through the MET channels presumed to be located at both ends of the stereocilia tip links (Denk et al., 1995). Also, the presence of PMCA2 in the basal portion of the stereocilia would isolate the MET channels from calcium fluctuations coming from the cell body. In fact, modeling the calcium clearance mechanism showed that, for the clearance mechanism to operate properly, high PMCA2 levels are required along the length of the stereocilia (Fettiplace and Ricci, 2005). The enrichment and uniform distribution of PMCA2 along the length of the stereocilia is also consistent with the recently proposed function of PMCA2 in maintaining elevated $\mathrm{Ca}^{2+}$ levels in the endolymph near the stereocilia bundles (Wood et al., 2004).

\section{PMCA2 mobility in stereocilia}

Our results on the clearance of PMCA2-antibody complexes indicates that PMCA2 molecules are continuously removed from stereocilia with an average half-life of residency time of $\sim 5 \mathrm{~h}$. We also show that newly synthesized PMCA2 molecules can be rapidly delivered to stereocilia. During the removal of PMCA2-antibody complexes or the incorporation of GFP-PMCA2 in stereocilia, we did not observe any clear front wave of migration. On the contrary, the stochastic pattern of distribution, the gradients from base to tip of stereocilia observed at the early times of GFPPMCA2 expression, and the kinetics of clearance of PMCA2antibody complexes are all consistent with a diffusion process. We observed that the global diffusion rate associated with the clearance process loosely matched the modeled clearance when using diffusion rates between 0.01 and $0.005 \mu \mathrm{m}^{2} / \mathrm{s}$. These values for the diffusion coefficient are at the lower end of the range commonly observed for the mobile fraction of plasma membrane proteins (Kusumi et al., 2005).

The relatively low estimated diffusion coefficient suggests that PMCA2 may be interacting transiently with some immobile or slowly moving component of stereocilia. We directly evaluated protein mobility in the membrane by studying the FRAP behavior of GFP-PMCA2 in stereocilia. FRAP experiments were performed on the two PMCA2 splice variants, PMCA2wa and PMCA2wb. Our results showed similar recovery behavior for both variants. For each splice form, we were able to distinguish two PMCA2 pools with distinct mobilities. The fraction that was able to rapidly diffuse, termed mobile fraction, corresponded to $\sim 60 \%$ of the total PMCA2 and showed a recovery profile consistent with a simple diffusion-based process. The diffusion coefficient estimated for this mobile fraction was between 0.1 and 0.2 $\mu \mathrm{m}^{2} / \mathrm{s}$. The remaining PMCA2 $(\sim 40 \%)$ failed to recover within the time course of our FRAP experiments. This stable pool contributes to the recruitment and enrichment of PMCA2 in the stereocilia. The stable pool is likely to result from complex formation through transient interactions of PMCA2 with more stationary components of the stereocilia.

\section{How is PMCA2 recruited to and maintained in stereocilia?}

The higher concentration of PMCA2 observed in stereocilia at steady state suggests a process of recruitment and maintenance. Maintenance of a 1.6-fold higher PMCA2 level in stereocilia compared with the apical plasma membrane at the base is likely related to the $\sim 40 \%$ immobile pool of PMCA2 that we observed in our FRAP experiments. Trapping of proteins into relatively immobile pools in stereocilia, as in other membranes, may depend on (1) interactions with underlying cytoskeleton and scaffolding proteins, (2) interactions with extracellular components, and/or (3) interactions in the plane of the membrane with specific lipid domains (Kusumi et al., 2005). Protein-protein contacts via the postsynaptic density-95/Discs large/zona occludens-1 (PDZ)binding domain are known to affect the stability and the plasma membrane subcompartment localization of proteins. PMCA4b, for example, is recruited to the actin cytoskeleton by interacting with PDZ domain-containing proteins; through this PDZbinding interaction, PMCA4b was shown to redistribute into 
newly formed filopodia in platelets (Dean and Whiteheart, 2004). Interestingly, we did not observe significant differences in the kinetics of incorporation into stereocilia and mobility between (1) GFP-PMCA2wb, which contains the PDZ-binding tail capable of establishing interaction with scaffolding proteins, and (2) GFP-PMCA2wa, which lacks the PDZ domain-interacting motif. This may suggest an absence of PDZ proteins available for interacting with PMCA2wb in stereocilia. An alternative possibility for the selective retention of PMCA2 in stereocilia is through its association with stable lipid subdomains (or lipid rafts), because there is abundant evidence that many proteins are dynamically associated with caveolae, or raft domains, in plasma membranes, including PMCA (Schnitzer et al., 1995; Pang et al., 2005).

\section{Adaptor proteins of the clathrin complex are excluded from stereocilia}

Previous studies have shown clathrin-coated pits and vesicles around the cuticular plate, suggesting that clathrin-mediated endocytosis is the major route for internalization of apical membrane proteins in hair cells. In our studies, we observed that endocytosis, formation of clathrin-coated pits, and the localization of adaptor proteins of the clathrin complex are all restricted to the pericuticular apical membrane and excluded from the stereocilia and kinocilia membrane. The molecular basis for the compartmentalization of PMCA2 and the components of the AP2 complex is unknown. One strategy appears to be the ability of specific compartments to exclude proteins. PMCA2 and components of the clathrin-mediated pathway, $\alpha$-adaptin and $\mu 2$, are constitutively excluded from the kinocilia membrane; moreover, overexpression of these proteins indicates that a highly effective filter limits diffusion of these proteins into the kinociliar compartment. This filter may lie at the base of the kinocilium in which multiple rings of closely associated protein particles form a structure called the "ciliary necklace" (Arima et al., 1986). In other cilia, this structure has been shown to separate membrane compartments (Montesano, 1979). The base of stereocilia is also not well characterized; in EM images, it appears shrouded by an electron-dense material that masks the tapered rootlets of stereocilia (Hackney and Furness, 1995). Therefore, it is possible that structural elements form a selective diffusion barrier, which allows PMCA2 proteins to pass but excludes $\alpha$-adaptin and $\mu 2$ subunits.

The localization of both endocytosis and exocytosis in close proximity to each other along a narrow band of apical plasma membrane near the cell border raises the question of how the cell minimizes the rapid futile recycling of newly synthesized proteins before they even reach their target in the stereocilia. The capture of protein cargo by adaptor proteins is likely to occur at any point of the apical cell surface excluding stereocilia and kinociliar membranes; however, completion of endocytosis appears to only occur at the periphery (Fig. 5). One possibility is that the association rate for $\mu 2$ binding to single PMCA2 molecules is significantly slower compared with the fast diffusion of PMCA2 throughout the apical membrane. On average, most of the newly delivered PMCA2 would therefore escape association with the AP2 complex and rapidly equilibrate throughout the stereocilia membrane.

\section{Implications of PMCA2 turnover for stereocilia plasticity and renewal}

Our results showing the constitutive removal of PMCA2 and rapid delivery and diffusion of GFP-PMCA2 to stereocilia sug- gests that PMCA2, like the actin core (Lin et al., 2005), is also rapidly turning over. A model for the apical recycling and enrichment of PMCA2 in the stereocilia is shown schematically in supplemental Figure 1 (available at www.jneurosci.org as supplemental material). The mechanisms for turnover and renewal of the actin core and the membrane proteins must be matched for a concerted dynamic regulation of the entire stereocilia bundle. Just as the hair cell must independently regulate actin addition at the tips and removal at the base of stereocilia, so too it must regulate the amount of new membrane insertion compared with the amount of endocytosis at the lateral margin of the hair cell to maintain stereocilia length and the steady-state levels of PMCA2 as well as other components of the stereocilia membrane.

\section{References}

Adato A, Michel V, Kikkawa Y, Reiners J, Alagramam KN, Weil D, Yonekawa H, Wolfrum U, El-Amraoui A, Petit C (2005) Interactions in the network of Usher syndrome type 1 proteins. Hum Mol Genet 14:347-356.

Andrews SS, Bray D (2004) Stochastic simulation of chemical reactions with spatial resolution and single molecule detail. Phys Biol 1:137-151.

Arima T, Masuda H, Uemura T (1986) Structural similarities between kinocilium of vestibular hair cell and tracheal motile cilium in the guinea pig. Auris Nasus Larynx 13 [Suppl 2]:S15-S19.

Chicka MC, Strehler EE (2003) Alternative splicing of the first intracellular loop of plasma membrane $\mathrm{Ca}^{2+}$-ATPase isoform 2 alters its membrane targeting. J Biol Chem 278:18464-18470.

Dean WL, Whiteheart SW (2004) Plasma membrane $\mathrm{Ca}^{2+}$-ATPase (PMCA) translocates to filopodia during platelet activation. Thromb Haemost 91:325-333.

DeMarco SJ, Strehler EE (2001) Plasma membrane $\mathrm{Ca}^{2+}$-atpase isoforms $2 \mathrm{~b}$ and $4 \mathrm{~b}$ interact promiscuously and selectively with members of the membrane-associated guanylate kinase family of PDZ (PSD95/Dlg/ ZO-1) domain-containing proteins. J Biol Chem 276:21594-21600.

DeMarco SJ, Chicka MC, Strehler EE (2002) Plasma membrane $\mathrm{Ca}^{2+} \mathrm{AT}$ Pase isoform $2 \mathrm{~b}$ interacts preferentially with $\mathrm{Na}^{+} / \mathrm{H}^{+}$exchanger regulatory factor 2 in apical plasma membranes. J Biol Chem 277:10506-10511.

Denk W, Holt JR, Shepherd GM, Corey DP (1995) Calcium imaging of single stereocilia in hair cells: localization of transduction channels at both ends of tip links. Neuron 15:1311-1321.

DeRosier DJ, Tilney LG (1989) The structure of the cuticular plate, an in vivo actin gel. J Cell Biol 109:2853-2867.

Dumont RA, Lins U, Filoteo AG, Penniston JT, Kachar B, Gillespie PG (2001) Plasma membrane $\mathrm{Ca}^{2+}$-ATPase isoform $2 \mathrm{a}$ is the PMCA of hair bundles. J Neurosci 21:5066-5078.

Fettiplace R, Ricci AJ (2005) Mechanoelectrical transduction in auditory hair cells. In: Vertebrate hair cells: Springer handbook of auditory research, Vol 27 (Eatock RA, Fay RR, Popper AN, eds). New York: Springer.

Gao WY, Ding DL, Zheng XY, Ruan FM, Liu YJ (1992) A comparison of changes in the stereocilia between temporary and permanent hearing losses in acoustic trauma. Hear Res 62:27-41.

Hackney CM, Furness DN (1995) Mechanotransduction in vertebrate hair cells: structure and function of the stereociliary bundle. Am J Physiol 268:C1-C13.

Jaeger RG, Fex J, Kachar B (1994) Structural basis for mechanical transduction in the frog vestibular sensory apparatus. II. The role of microtubules in the organization of the cuticular plate. Hear Res 77:207-215.

Kachar B, Battaglia A, Fex J (1997) Compartmentalized vesicular traffic around the hair cell cuticular plate. Hear Res 107:102-112.

Kachar B, Parakkal M, Kurc M, Zhao Y, Gillespie PG (2000) Highresolution structure of hair-cell tip links. Proc Natl Acad Sci USA 97:13336-13341.

Kozel PJ, Davis RR, Krieg EF, Shull GE, Erway LC (2002) Deficiency in plasma membrane calcium ATPase isoform 2 increases susceptibility to noise-induced hearing loss in mice. Hear Res 164:231-239.

Kusumi A, Nakada C, Ritchie K, Murase K, Suzuki K, Murakoshi H, Kasai RS, Kondo J, Fujiwara T (2005) Paradigm shift of the plasma membrane concept from the two-dimensional continuum fluid to the partitioned fluid: high-speed single-molecule tracking of membrane molecules. Annu Rev Biophys Biomol Struct 34:351-378.

LeMasurier M, Gillespie PG (2005) Hair-cell mechanotransduction and cochlear amplification. Neuron 48:403-415. 
Lin HW, Schneider ME, Kachar B (2005) When size matters: the dynamic regulation of stereocilia lengths. Curr Opin Cell Biol 17:55-61.

Lipkow K, Andrews SS, Bray D (2005) Simulated diffusion of phosphorylated CheY through the cytoplasm of Escherichia coli. J Bacteriol 187:45-53.

Lumpkin EA, Hudspeth AJ (1998) Regulation of free $\mathrm{Ca}^{2+}$ concentration in hair-cell stereocilia. J Neurosci 18:6300-6318.

Montesano R (1979) Inhomogeneous distribution of filipin-sterol complexes in the ciliary membrane of rat tracheal epithelium. Am J Anat 156:139-145

Nakatsu F, Ohno H (2003) Adaptor protein complexes as the key regulators of protein sorting in the post-Golgi network. Cell Struct Funct 28:419-429.

Pang Y, Zhu H, Wu P, Chen J (2005) The characterization of plasma membrane $\mathrm{Ca}^{2+}$-ATPase in rich sphingomyelin-cholesterol domains. FEBS Lett 579:2397-2403.

Rzadzinska AK, Schneider ME, Davies C, Riordan GP, Kachar B (2004) An actin molecular treadmill and myosins maintain stereocilia functional architecture and self-renewal. J Cell Biol 164:887-897.

Saxton MJ, Jacobson K (1997) Single-particle tracking: applications to membrane dynamics. Annu Rev Biophys Biomol Struct 26:373-399.
Schneider ME, Belyantseva IA, Azevedo RB, Kachar B (2002) Rapid renewal of auditory hair bundles. Nature 418:837-838.

Schnitzer JE, Oh P, Jacobson BS, Dvorak AM (1995) Caveolae from luminal plasmalemma of rat lung endothelium: microdomains enriched in caveolin, $\mathrm{Ca}^{2+}$-ATPase, and inositol trisphosphate receptor. Proc Natl Acad Sci USA 92:1759-1763.

Street VA, McKee-Johnson JW, Fonseca RC, Tempel BL, Noben-Trauth K (1998) Mutations in a plasma membrane $\mathrm{Ca}^{2+}$-ATPase gene cause deafness in deafwaddler mice. Nat Genet 19:390-394.

Strehler EE, Zacharias DA (2001) Role of alternative splicing in generating isoform diversity among plasma membrane calcium pumps. Physiol Rev 81:21-50.

Wood JD, Muchinsky SJ, Filoteo AG, Penniston JT, Tempel BL (2004) Low endolymph calcium concentrations in deafwaddler $2 \mathrm{~J}$ mice suggest that PMCA2 contributes to endolymph calcium maintenance. J Assoc Res Otolaryngol 5:99-110.

Wu YC, Ricci AJ, Fettiplace R (1999) Two components of transducer adaptation in auditory hair cells. J Neurophysiol 82:2171-2181.

Yamoah EN, Lumpkin EA, Dumont RA, Smith PJ, Hudspeth AJ, Gillespie PG (1998) Plasma membrane $\mathrm{Ca}^{2+}$-ATPase extrudes $\mathrm{Ca}^{2+}$ from hair cell stereocilia. J Neurosci 18:610-624. 